\title{
Contracampo
}

\section{Variações sobre o uso do Skype na pesquisa empírica em comunicação: apontamentos metodológicos}

\author{
Variations on the use of Skype \\ in the empirical research in Communication: \\ methodological notes
}

Adriana Braga

adrianabraga1@yahoo.com.br

Professora no Programa de Pós Graduação em Comunicação da PUC-Rio e Pesquisadora do CNPq na área de Comunicação. Autora dos livros "CMC. Identidades e Género: teoria e método" (Portugal, Editora da UBI, 2005) e "Personas Materno-

Eletrônicas" (Porto Alegre, Editora Sulina, 2008).

Édison Gastaldo edisongastaldo@yahoo.com.br

Professor no Programa de Pós Graduação em Ciências Sociais da UFRRJ e Pesquisador do CNPq na área de Antropologia. Autor dos livros "Pátria, Chuteiras e Propaganda" (AnnaBlume/Unisinos, 2002), "Erving Goffman, desbravador do cotidiano" (Tomo Editorial, 2004) e "Nações em Campo" (com Simoni Guedes, Intertexto, 2006.:

Uma versão prévia deste artigo foi apresentada ao Grupo de Trabalho "Recepção: processos interpretativos, usos e consumos midiáticos", do XX Encontro da Compós, na UFRGS, Porto Alegre, RS, em junho de 2011.

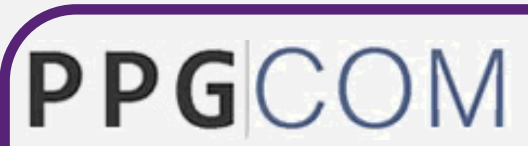
UFF

Ao citar este artigo, utilize a seguinte referência bibliográfica

BRAGA, Adriana; GASTALDO, Édison. Variações sobre o uso do Skype na pesquisa empírica em comunicação: apontamentos metodológicos. In: Revista Contracampo, v. 24, n. 1, ed. julho, ano 2012. Niterói: Contracampo, 2012. Pags: 04-18.

Enviado em: 12 de fev. de 2012 Aceito em: 16 de jul. De 2012

\section{Edição 24/2012}

Ensaio temático "Sociabilidades em rede"

Contracampo

e-ISSN 2238-2577

Niterói (RJ), v. 24, n. 1, jul./2012.

www.uff.br/contracampo

A Revista Contracampo é uma revista eletrônica do Programa de PósGraduação em Comunicação da Universidade Federal Fluminense e tem como objetivo contribuir para a reflexão crítica em torno do campo midiático, atuando como espaço de circulação da pesquisa e do pensamento acadêmico. 


\section{Resumo}

Este artigo busca apresentar e discutir aspectos da utilização do aplicativo Skype para a pesquisa empírica em Comunicação. O Skype é um programa gratuito (freeware) que permite conversações simultâneas em áudio e vídeo usando computadores. A reflexão acerca da comunicação interpessoal mediada tem há mais de um século o telefone - voz sem imagem - como referência, embora a ideia do "videofone" já esteja presente desde os primórdios da televisão, em filmes de ação ou ficção científica. A introdução de um canal de vídeo propõe uma série de desafios teóricos e metodológicos para a pesquisa em Comunicação. Por exemplo, uma conversação por Skype poderia ser considerada uma "interação face a face"? $\mathrm{O}$ artigo discute também o uso de algumas potencialidades desta tecnologia na pesquisa científica, como objeto de investigação, como meio técnico de registro de dados e como ferramenta analítica.

Palavras-chave: Interação social; Comunicação Mediada por Computador; Metodologia de Pesquisa; Skype.

\section{Abstract}

This paper seeks to present and discuss some Skype features applied to empirical research on communications. Skype is a free software that allows simultaneous conversation using audio and video between computers. Mediated interpersonal communications have had the telephone - voice without image - as its reference for over a century, although the idea of the 'videophone' have been present since the beginning of television in science fiction movies. The introduction of a video channel poses several theoretical and methodological challenges to communication researchers. For instance, can a Skype conversation be considered 'face-to-face interaction'? This paper also discusses the use of some possibilities of this technology on scientific research, as an object of enquiry, as a medium of recording data and as analytical tool.

Keywords: Social Interaction; Computer Mediated Communication; Research Methodology; Skype. 
$\mathrm{E}$

ste artigo busca apresentar e discutir alguns aspectos da utilização do aplicativo "Skype”, programa de distribuição gratuita (freeware) que permite conversações simultâneas de áudio e vídeo através de computadores, além de teleconferências, ligações de e para telefones fixos e celulares, entre outros recursos. A reflexão acerca da comunicação interpessoal mediada tecnologicamente tem há mais de um século o telefone - um meio que permite a transmissão da voz, mas não da imagem - como sua principal referência. Não obstante, desde pelo menos os anos 1930, quando a televisão começou a ser comercializada nos EUA, a ideia de um "videofone", transmitindo imagem e som em comunicações interpessoais tem sido representada em filmes e histórias em quadrinhos, como Dick Tracy, de Chester Gould (1931) e Flash Gordon, de Alex Raymond (1934), personagens que se comunicam com seus colegas usando um videofone de pulso, em enredos futuristas ou de "ficção científica".

Assim, a realização da fantasia de décadas passadas resulta da combinação entre avanço tecnológico, simplicidade e distribuição gratuita. O Skype, juntamente com outros provedores de tecnologia VoIP (Voice over Internet Protocol), vêm contemporaneamente redefinindo a noção de comunicação telefônica em todo o mundo.

Considerando que, apesar das potencialidades técnicas trazidas pela Internet serem conhecidas, elas ainda são largamente subutilizadas na pesquisa científica, que continua sendo um meio essencialmente baseado em texto, e que, como tal, prioriza o uso da Internet para pesquisa documental - escrita - e em questionários enviados por email. A popularização de uma tecnologia como o Skype permite que se trabalhe digitalmente com dados, técnicas e métodos ligados à pesquisa qualitativa com som e imagem, oferecendo dados de contexto que permitem interpretações densas de fenômenos sociais, revitalizando a perspectiva etnográfica, de história oral e os estudos de recepção.

Neste artigo, queremos endereçar algumas questões relativas à introdução do canal de vídeo na comunicação mediada interpessoal e seus reflexos na pesquisa empírica em comunicação. Após uma breve apresentação da questão metodológica central e de uma caracterização do aplicativo Skype, discutimos as implicações teóricas da introdução desta tecnologia aos estudos da interação face a face, bem como alguns de 
seus desdobramentos na pesquisa empírica em Comunicação, tomando-a tanto como objeto de investigação, como meio técnico de registro de dados e como ferramenta analítica.

\section{Uma discussão metodológica}

Em seu inspirado texto intitulado "Sobre Metodologia", Howard Becker incentiva pesquisadores/as de campo a produzirem reflexões teórico-metodológicas originais, argumentando que "a metodologia é importante demais para ser deixada aos metodólogos" (BECKER, 1998, p. 17). No seu caso, Becker criticava o desenvolvimento de uma especialidade no contexto da Sociologia americana, que, ao invés de tentar lidar com e resolver problemas reais, oriundos de pesquisas empíricas, tentava normatizar os processos da produção de conhecimento científico alheio, particularmente na chamada Sociologia Quantitativa, com suas técnicas de mensuração como Surveys e Análise de Variáveis, que, em meados do século XX, começavam a dominar a ciência social norteamericana.

Em nosso caso, acreditamos que seja importante para pesquisadores/as que trabalham usualmente com objetos empíricos específicos colaborarem com a produção de reflexão teórica acerca dos desafios metodológicos que enfrentam. A inspiração para o título e para a produção deste artigo vem do clássico livro Variações sobre a técnica de gravador no registro da informação viva, de Maria Isaura Pereira de Queiroz (1991). Neste livro, infelizmente esgotado, Queiroz propõe uma discussão a propósito das implicações da possibilidade técnica de gravar depoimentos para posterior transcrição. Por assim dizer, a introdução do gravador possibilitou o pleno desenvolvimento de uma importante vertente metodológica da pesquisa em ciências humanas e sociais: as histórias de vida ou história oral. Da mesma maneira, acreditamos que a introdução de um canal de vídeo em um meio de comunicação interpessoal traz uma série de consequências não apenas para a vida cotidiana de quem o utiliza, mas também para a pesquisa que adota este meio como instrumento de trabalho ou objeto de investigação. A rápida popularização do aplicativo Skype coloca-o como um elemento fundamental para compreender as novas configurações da midiatização das relações interpessoais na contemporaneidade, motivo que nos leva a propor esta reflexão. 


\section{Sobre o Skype}

O Skype é um aplicativo para computadores que combina tecnologia VoIP com a organização de uma base de dados de participantes, aparentada com as chamadas redes sociais. Criada em 2003, a empresa que fundamentou sua popularidade na distribuição gratuita pela Internet de seu sistema VoIP foi vendida dois anos depois para o site $e$-Bay por uma soma estimada em 2,6 bilhões de dólares (fonte: www.skype.com ). Segundo uma estimativa da empresa, em 2009, o Skype contava com 540 milhões de participantes cadastrados em todo o mundo (no mesmo período, o Facebook, contava com 400 milhões de membros. Recentemente, foi estabelecido um recorde de conexão simultânea, em um total de 27 milhões de pessoas.

A tecnologia VoIP consiste em um desenvolvimento relativamente recente da informática, que foi viabilizado a partir da popularização das conexões domésticas em sistema banda larga (DSL), no início dos anos 2000. O termo VoIP é uma sigla que significa Voice Over Internet Protocol. O website How Stuff Works dá a seguinte definição, singela mas precisa: "VoIP é um método para tomar sinais de áudio analógico, como os que você ouve quando fala ao telefone, e transformá-los em dados digitais que podem ser transmitidos pela Internet” (HOW STUFF WORKS). ${ }^{1}$

Atualmente, o Skype permite que se façam chamadas usando o microfone e as caixas de som de um computador, ligando tanto para outros computadores conectados quanto para telefones fixos e móveis do mundo todo, usando o sistema SkypeOut. Para isto, é preciso que se comprem créditos telefônicos usando cartão de crédito e se podem fazer chamadas nacionais e internacionais a preços inferiores a qualquer operadora convencional $^{2}$. Além de sistema de mensageiro instantâneo (conveniente em caso de conexão fraca), o aplicativo ainda permite teleconferências - somente com voz conectando até 100 pessoas simultaneamente ou videoconferências com até 10 simultaneamente, embora o Skype recomende que videoconferências sejam de cinco pessoas ou menos, para não haver perda de qualidade de transmissão.

\footnotetext{
1 Tradução pessoal. No original: "Voice over Internet Protocol is a method for taking analog audio signals, like the kind you hear when you talk on the phone, and turning them into digital data that can be transmitted over the Internet."

${ }^{2}$ Por exemplo, 1 centavo por minuto de qualquer lugar do mundo para os EUA.
} 
Para entrar no sistema, é preciso instalar o programa Skype.exe, disponível gratuitamente no website www.skype.com e preencher um cadastro, criando um nome de acesso, uma senha e informando alguns dados mínimos (nome, cidade, sexo, idade etc.), mas que não são obrigatórios. Com esse cadastro, pode-se navegar entre os milhões de perfis, como em qualquer rede social. Pode-se solicitar a uma pessoa que se permita falar com ela. Caso se obtenha autorização, esta pessoa passará a constar na lista de contatos, indicando seu status de conexão - ausente, disponível, ocupado etc.

Em si, o Skype não traz nenhum grande avanço tecnológico que lhe seja exclusivo. Mas vem conseguindo concretizar a ficção do videofone ao combinar simplicidade de uso e custo baixo (desde que se disponha previamente de um computador com microfone, placa de som, alto-falantes e acesso à Internet em banda larga). Assim, de todas as muitas possibilidades deste meio de comunicação relativamente novo, queremos concentrar nossa atenção na conversação com áudio e vídeo. Atualmente, isso é realizado majoritariamente entre computadores, embora a recente adaptação do aplicativo para telefones móveis do tipo smartphone permita que essas conversações mediadas por áudio e vídeo possam ser bastante popularizadas em futuro próximo.

\section{Skype e interação face a face}

Podemos começar este tópico retomando a questão proposta no resumo: pode-se considerar interação simultânea mediada por áudio e vídeo como "interação face a face"? A questão é capciosa, pois literalmente, as pessoas participantes estão vendo a face uma da outra. Não obstante, elas não compartilham o mesmo ambiente físico. Ainda assim, trata-se de uma interação distinta e incomparavelmente mais rica em informação do que um telefonema ou uma troca de e-mails.

Para explorar esta questão, vamos iniciar definindo alguns termos, segundo a perspectiva teórica de Erving Goffman, talvez o autor que maior atenção dedicou aos elementos formais da interação social, o domínio do conhecimento a que chamou de Ordem da Interação. Em seu livro de 1967, Interaction Ritual, Goffman afirmou que o estudo das interações sociais em situação natural ainda não tinha um nome, mas que certamente já tinha um objeto: 
É a classe de eventos que ocorre durante a co-presença e por efeito da co-presença. Os materiais comportamentais finais são os olhares, os gestos, posicionamentos e declarações verbais que as pessoas continuamente colocam na situação, intencionalmente ou não. São sinais exteriores de orientação e envolvimento - estados de corpo e mente que não são normalmente investigados a propósito de sua organização social (...) Um dos objetivos ao lidar com estes dados é descrever as unidades naturais da interação das quais eles são feitos, começando com os menores - por exemplo, os fugazes movimentos faciais que um indivíduo pode fazer no jogo de expressar seu alinhamento ao que está ocorrendo - e terminando com temas como congressos de uma semana, sendo estes os mastodontes interacionais que levam ao limite aquilo que possa ser chamado de ocasião social. (GOFFMAN, 1967, p.1) ${ }^{3}$

É interessante destacar que todos os exemplos trazidos por Goffman de materiais significantes em termos interacionais estão disponíveis aos participantes de uma interação mediada por áudio e vídeo. Embora um aplicativo como o Skype - bem como computadores pessoais - fossem, ao tempo de Goffman, apenas fantasia futurista. É evidente que uma série de elementos da interação face a face, considerada nestes termos, não ocorre no Skype, como a co-presença física imediata. Elementos interacionalmente significantes, como cheiros, tremores de mãos, suores, vestimentas ou posicionamento corporal não estão acessíveis ou estão apenas parcialmente em uma interação mediada por Skype, na medida em que o plano das tomadas de câmera normalmente fica entre o que em cinema se chamaria um "plano médio fechado" e um "close-up".

Para discutirmos se a interação mediada por Skype pode ser considerada uma interação face a face, é importante definirmos o que entendemos por face. A noção de face, nos termos de Goffman, refere-se a uma dimensão moral do self, e é definida como sendo "o valor social positivo que uma pessoa efetivamente reivindica para si (...), é uma imagem do self delineada em termos de atributos sociais aprovados". (GOFFMAN,

\footnotetext{
${ }^{3}$ Tradução pessoal. No original: It is that class of events which occurs during co-presence and by virtue of co-presence. The ultimate behavioral materials are the glances, gesture, positionings and verbal statements that people continuously feed into the situation, whether intended or not. These are the external signs of orientation and involvement - states of mind and body not ordinarily examined with respect to their social organization. (...)One objective in dealing with these data is to describe the natural units of interaction build up from them, beginning with the littlest - for example, the fleeting facial move an individual can make in the game of expressing his allignement to what is happening - and ending with affairs such as week-long conferences, these being the interactional mastodons that push to the limit what can be called a social occasion (GOFFMAN, 1967, p. 1).
} 
1967, p.5) Assim, cada pessoa em interação com outras tem um conjunto de definições valorativas acerca de si mesmo para defender, e de prerrogativas a serem reconhecidas. A toda esta "tarefa" de manter-se à altura da própria dignidade, Goffman denomina facework, ou "trabalho de face". É uma categoria interessante para pensar que, na dinâmica de situações sociais que as pesquisas naturalistas buscam apreender, existem forças sociais atuando na conduta dos indivíduos, que os fazem atuar ativamente em prol da defesa de sua face. Goffman aponta uma série de ações relativas ao trabalho de face, como evitar lugares, pessoas e situações que possam por em risco a face a ser defendida, ou o uso de expressões rituais de interação: "com licença", "desculpe", "obrigado" etc.

Ora, considerando a face como uma entidade moral (muito além de um rosto), a maior parte dos elementos componentes do trabalho de face podem ser não apenas presenciados, mas também gravados e analisados se a interação for mediada pelo Skype. Mesmo que não haja co-presença stricto sensu, ainda assim existe nestas interações tecnologicamente mediadas um ganho substancial em qualidade e sutileza dos dados para quem lida com qualquer tipo de entrevista na pesquisa empírica, como veremos a seguir.

Outro ponto que merece destaque nesta discussão é um aspecto central da interação face a face: o olhar. Em uma interação presencial, boa parte da definição de uma situação se expressa na troca dos olhares. De quem olha para o alto, em súplica, a quem deliberadamente evita o olhar do outro, "virando a cara", a troca de olhares entre os participantes é parte fundamental de qualquer interação face a face. Entretanto, no Skype, a exemplo de qualquer interação através de uma webcam, a virtual impossibilidade de contato com o olhar do outro fica rapidamente evidente.

$\mathrm{Na}$ interação face a face, olha-se diretamente - sem mediação - nos olhos do outro. No Skype, o que cada participante vê é a imagem que a câmera transmite do outro. Como, por uma diferença de alguns centímetros (o termo técnico é denominado "erro de Paralaxe"), a tela para a qual se olha está distante do eixo ótico da lente, a imagem que cada um vê do outro ao olhar a tela é uma imagem sem contato entre os olhares. Como se cada um falasse com uma pessoa ocupada em outra coisa. Paradoxalmente, a única maneira de olhar diretamente nos olhos do outro é fitar a lente. 
Ao fazê-lo, o próprio sujeito perde o foco na imagem do outro, que fica reduzida à visão periférica.

Curiosamente, a única maneira de conseguir superar esta aparente impossibilidade é contar com a falha tecnológica: por vezes, a conexão faz as imagens congelarem ou atrasarem por algumas frações de segundo, o suficiente para que se possa ver, num relance, uma imagem similar à presencial, apesar de ser, no limite, um instante do passado.

No canto da imagem existe um outro componente importante de trazer à discussão. Trata-se de uma pequena tela com a imagem da câmera de cada participante. Este elemento de feedback visual funciona como uma espécie de espelho, onde se tem acesso à imagem que o outro recebe, e que é um recurso fundamental no que Goffman chama de "manejo da impressão" (GOFFMAN, 1998). Poderíamos chamar a esta preocupação com o que o outro verá de "controle do quadro". Na medida em que a fração do real que pode ser digitalizada e transmitida via Skype se reduz a um quadro retangular em proporção 3 x 4 horizontal, podemos considerar esta área como "palco", e o espaço off em torno a este quadro como "bastidores" (v. GOFFMAN, 1998). A janela de feedback é fundamental para corrigir problemas prosaicos como reflexos indesejados em óculos, um cabelo despenteado ou uma camisa desarrumada ${ }^{4}$, mas também serve para microajustes - manejo - da impressão. No Skype, se tem acesso a uma visão privilegiadíssima de si, que permite "monitorar" a cada momento a expressão mais adequada ao que se está dizendo. Como alguém que, ao falar com os outros, tivesse sempre diante de si um espelho. E com o descompromisso - mais que isso, a impossibilidade - de olhar nos olhos do outro. Uma considerável diferença para situações de encontro face a face.

\section{O Skype na Pesquisa}

Neste tópico, endereçaremos alguns pontos para discussão acerca das potencialidades do Skype na pesquisa científica: a) como objeto de investigação de per se; b) como ferramenta de coleta e sistematização de dados; e c) como ferramenta analítica.

\footnotetext{
${ }^{4}$ Ou a clássica gag de comédias pastelão, de filmar um personagem em frente a uma cabeça de touro empalhada na parede, e com isso, dar-lhe "chifres".
} 
Como objeto de investigação científica, a Plataforma Skype e sua combinação de rede social com aplicativo VoIP simplificado permite uma ampla gama de abordagens empíricas. Os metrics ${ }^{5}$ disponíveis no Skype permitem uma série de estudos, relacionando dados como a dispersão geográfica de participantes on-line (cada endereço IP tem uma localização geográfica específica), distribuição por gênero, por idade, profissão e imagem pessoal declaradas etc. A análise destas variáveis quantitativas já permite, por si só, uma multiplicidade de caminhos para a pesquisa. Em uma perspectiva etnográfica, o aplicativo Skype pode ser pesquisado em termos de seus usos concretos, em situações específicas, ou seja, sobre o que pessoas fazem com o aplicativo em suas vidas cotidianas. Para tanto, gravações em vídeo das situações naturais de uso do aplicativo podem ser boas ferramentas para análise deste fenômeno do ponto de vista dos sujeitos, o que evidentemente não dispensa o uso concomitante de técnicas de pesquisa mais tradicionais, como a observação participante e o diário de campo etnográfico. Trataremos mais desse aspecto de registro de dados a seguir. Outra vertente do fenômeno com bom potencial a ser explorado consiste na análise das apresentações do self de participantes do Skype, elaborada a partir dos perfis publicados no aplicativo. Com que termos os/as participantes se apresentam, que imagem de si escolhem para exibir publicamente, qual a lógica empregada na formulação do "nome Skype" ou mesmo que campos do formulário não foram preenchidos são todos dimensões significantes deste fenômeno.

Na utilização do Skype como meio de registro de dados de pesquisa, é importante destacar a pertinência e a atualidade da reflexão de Queiroz (1991) acerca da gravação e transcrição de entrevistas - ou, em seus termos, "informação viva". Afinal, ao se obter uma entrevista gravada, seja em meio digital ou magnético, com ou sem imagem ${ }^{6}$, existem os mesmos riscos de bias, de condução do entrevistado/a pelo entrevistador/a, bem como as mesmas - inevitáveis - mediações entre a fala gravada e o documento escrito. O que acreditamos ser necessário enfatizar é que no Skype, uma comunicação interpessoal pode se dar tanto por texto escrito (mensageiro instantâneo), por som (telefone), por som e imagem (videofone) ou por qualquer combinação dos três meios.

\footnotetext{
5 Dados quantitativos gerados automaticamente por programas de computador e que podem ser armazenados e contabilizados.

${ }^{6} \mathrm{O}$ uso de câmeras de fotografia e cinema como ferramenta de pesquisa na antropologia é quase tão antigo como a invenção destes meios. Franz Boas, Bronislaw Malinowski e Robert Flaherty, entre outros, foram pioneiros desta nascente "antropologia visual” já nos primeiros anos do século XX.
} 
Um diálogo através do mensageiro instantâneo tem seus próprios ritmos e peculiaridades, diferentes, por exemplo, de uma troca de bilhetes ou cartas, como a peculiar alternância de enunciador, muito bem discutida em Greiffenhagen \& Watson (2005). ${ }^{7}$ Cada um destes meios tem suas próprias especificidades no que diz respeito a armazenamento e organização de arquivos de dados. As mensagens escritas, por exemplo, são facilmente organizáveis e repletas de detalhamentos úteis para a pesquisa. Cada enunciado vem acompanhado de uma legenda com a data, a hora e o nome do enunciador, bastando selecionar o trecho desejado, copiar e colar em um processador de texto para se ter já transcrito e em arquivo uma interação verbal. Pode-se obter conversas antigas, reunindo com facilidade um grande volume de dados. Muitas vezes, como disseram Rutter e Smith (2005, p. 84), “pesquisa on-line é uma espécie de sonho do pesquisador". Esta parece ser uma delas.

Já com relação ao áudio, existe uma série de programas que permitem capturar o áudio de uma conversa ou entrevista via Skype. Neste caso, o procedimento torna-se bastante similar ao de um gravador magnético, porém com as facilidades de uma mídia digital. Um arquivo de áudio digital pode ser copiado, enviado por e-mail, gravado em um $\mathrm{CD}$ ou pendrive etc. Assim, distribuir uma gravação para, por exemplo, participantes de um grupo de pesquisa permite que a tarefa de transcrição possa ser dividida, ou feita coletivamente, o que dificilmente ocorreria com uma matriz em fita magnética.

O passo mais difícil é o de conseguir gravar uma interação em videofone. Isso se torna mais contrastante quando se percebe o quanto é simples realizar uma interação em videofone por Skype. Em consulta ao forum de discussão disponibilizado pela Cia. Skype, pode-se perceber o quanto esta possibilidade técnica, apesar de existir, é ainda complexa e restrita a participantes experientes. Várias pessoas reclamam de algum dos seguintes problemas: gravação da imagem de um só dos computadores; gravação de imagens, mas sem som; muito baixa qualidade de imagem; ausência de sincronismo entre áudio e vídeo; pane pura e simples. Vários outros participantes do fórum recomendam diferentes aplicativos para tentar resolver o problema. O fato de cada pessoa recomendar um aplicativo diferente é um indicador da ausência de consenso

\footnotetext{
${ }^{7}$ Para uma boa discussão acerca das especificidades da atividade online em mensageiros instantâneos, ver também Braga, 2008.
} 
quanto à melhor opção - ou mesmo se há alguma opção melhor. Um dos depoentes deixou a seguinte mensagem, que exemplifica bem este ponto:

\begin{abstract}
Alguém+: eu me perguntava porque a Cia Skype ainda nos tortura assim, eles simplesmente deveriam incluir isso [a gravação em audio e video] no Skype... é tããããão fácil! Nós não estamos pedindo a eles para inventarem a nave do Star Trek! Da minha experiência, eu só posso alertar o resto dos colegas para usarem CamStudio [um aplicativo de gravação e edição de video] para gravar o video que entra, mas será em baixa qualidade e sem som! $<<<$ espero que o Skype do ano 2067 consiga fazer isso! >> (SKYPE COMMUNITY) ${ }^{8}$
\end{abstract}

O exemplo é interessante para evidenciar tanto as dificuldades técnicas que ainda existem para realizar gravações de conversas com áudio e vídeo quanto a dimensão de "obra em processo" do próprio aplicativo, que ainda está longe da perfeição.

Uma alternativa para minimizar o problema pode ser a gravação das interações em uma câmera de vídeo convencional, digital ou analógica, como sugerimos para pesquisas etnográficas. Problemas técnicos podem decorrer, entretanto, pela sincronicidade da gravação da câmera com a da reprodução da imagem em movimento pelo computador, resultando nos chamados "batimentos", faixas pretas horizontais que aparecem na tela que está sendo filmada. A gravação em uma câmera de vídeo tem, por outro lado, a vantagem de permitir uma visada mais ampla sobre a situação na qual a interação ocorre, para além do campo rigidamente demarcado pela webcam.

De qualquer maneira, a gravação das conversas com imagem e som, mesmo que ainda seja problemática, não implica que não se deva explorar toda a sua riqueza e potencial analítico. Caso se consiga gravar ao menos o áudio da conversa para posterior transcrição, a elaboração de um diário de campo relatando sistematicamente a experiência pode ajudar a cobrir as lacunas deixadas pelos limites do meio técnico.

Finalmente, gostaríamos agora de pensar sobre o uso do Skype como ferramenta analítica, isto é, como se podem analisar os conteúdos de interações possibilitadas por este meio. Em primeiro lugar, é importante distinguir os diferentes tipos de dados passíveis de coleta neste aplicativo. Os indicadores quantitativos (metrics, mencionados

\footnotetext{
${ }^{8}$ Tradução pessoal. No original:

"Someone+: I am wondering why Skype's company still torture us like this, they must just to include that in Skype,,,it sooo easy!We do not require from them to invent the Star Trek ship! From my experience I can only advice the rest guys to use CamStudio to records the incoming video but it will be with low quality and without sound! $<<<$ I will hope the Skype from 2067 year to be able to do this! >> "
} 
acima) podem ser organizados em planilhas de base de dados, permitindo que se trabalhe com análise de variáveis, embora assumindo-se de antemão que as informações oferecidas nos formulários de perfil não são completamente confiáveis.

Os dados de texto, como os de mensageiro instantâneo, podem ser objeto de uma Análise de Discurso, buscando na fala de participantes as lógicas simbólicas, hierarquizações e marcas de fundo ideológico de seus contextos de produção discursiva.

As conversas com voz permitem a realização de entrevistas de todos os tipos, com informantes espalhados pelo mundo. Aqui, talvez o maior problema seja justamente a seleção de informantes.

Imagens gravadas de videofone ou videoconferência podem ser uma extraordinária fonte de dados para pesquisas sobre interação social midiatizada. Em uma situação de pesquisa (por exemplo, na gravação de um vídeo com entrevistas), não se coloca nenhuma pessoa em frente a uma câmera para gravar um depoimento sem uma série de negociações prévias, e sem superar uma série de embaraços e constrangimentos. No Skype, a presença da câmera é condição necessária à própria existência da interação, faz parte do acordo tácito que precede o uso do aplicativo: só se pode ver e ser visto através das câmeras.

Em comparação, em uma situação social natural, a entrada em cena de uma câmera de vídeo torna-a basicamente uma "intrusa". A vida cotidiana - supostamente não está sendo filmada (embora a multiplicidade e a dispersão de câmeras de vigilância não permita muita certeza sobre isso). Ou seja, previamente a qualquer filmagem em situação de campo, é preciso certo tempo de contato e certo trabalho de negociação com os/as participantes. Interações em vídeo e áudio gravadas no Skype não eximem o/a pesquisador/a de negociar previamente com informantes ou sujeitos da pesquisa, mas o embaraço situacional decorrente da presença da câmara fica certamente minimizado.

\section{Conclusão}

Neste artigo, buscamos colocar em questão alguns aspectos teóricos e metodológicos da utilização do aplicativo Skype na pesquisa científica em Comunicação. Não obstante o foco disciplinar, trata-se de uma discussão metodologicamente relevante para todas as áreas que trabalham com abordagens 
empíricas qualitativas (particularmente entrevistas), como as Ciências Sociais, Psicologia, História, Educação etc.

A possibilidade de interação com os/as sujeitos de pesquisa é muitas vezes determinante no sucesso de uma investigação em ciências humanas e sociais. Se uma interação mediada tecnologicamente na qual os interagentes podem ver suas imagens e ouvir suas vozes simultaneamente está longe de se constituir em uma situação de copresença física imediata, por outro lado, está muito acima - em sutileza e riqueza de informação relevante - de uma entrevista gravada, por telefone ou por e-mail. Assim, explorar as múltiplas possibilidades de um meio técnico traz vantagens de várias ordens para a pesquisa científica. Desde as mais prosaicas, como permitir reuniões de grupos de pesquisa, bancas ou encontros de trabalho dispersos nacional e internacionalmente, até a atividade mais diretamente de pesquisa, seja como objeto de investigação ou meio técnico de coleta, sistematização e análise de dados.

Considerando o imenso potencial destes meios, chega a parecer um recuo que tantas pesquisas em Comunicação usem questionários enviados por e-mail como principal fonte de dados, subestimando algumas excepcionais possibilidades desse instrumento de interação e obtenção de dados na pesquisa empírica. Ao reduzir a interação entre pesquisadores/as e pesquisados/as à troca de mensagens escritas, perdese todo o potencial analítico de uma situação natural. A complexidade do mundo social vista pelos olhos do outro, tão bem sintetizada na expressão “informação viva" é descartada e o fenômeno, muitas vezes, permanece incompreendido. Este texto busca estimular a descoberta e exploração destes novos caminhos, que ajudem a redescobrir o lado vivo da pesquisa.

\section{Referências Bibliográficas}

BECKER, Howard. Métodos de Pesquisa em Ciências Sociais. São Paulo: Hucitec, 1998.

BRAGA, Adriana. Personas materno-eletrônicas: interação social no blog Mothern. Porto Alegre: Sulina, 2008.

GOFFMAN, Erving. Interaction Ritual: essays on face-to-face behavior. New York: Anchor Books, 1967.

A representação do Eu na vida cotidiana. Petrópolis: Vozes, 1998. 
GREIFFENHAGEN, Christian \& WATSON, Rod. "Teoria' e 'Método' na CMC: identidade, gênero e tomada-de-turno: uma abordagem etnometodológica e analítico-conversacional". In: BRAGA, Adriana (org.) CMC, Identidades e Género: teoria e método. Covilhã, Portugal: Editora da UBI, 2005.

RUTTER, Jason \& SMITH, Greg. "Etnographic Presence in Nebulous Settings.” In: HINE, Chritine. Virtual Methods: Issues in Social Research on the Internet. Oxford: Berg Publishers, 2005.

QUEIROZ, Maria Isaura Pereira de. Variações sobre a técnica de gravador no registro da informação viva. São Paulo: T.A. Queiroz, 1991.

Websites:

How Stuff Works: www.howstuffworks.com

Skype: www.skype.com

Skype community: http://forum.skype.com/index.php?showtopic=52297 